\title{
RUPTURA ISOLADA DA PELVE RENAL SECUNDÁRIA AO TRAUMA ABDOMINAL CONTUSO
}

\section{ISOLATED DISRUPTION OF THE RENAL PELVIS BY BLUNT TRAUMA}

\section{Sizenando Vieira Starling, TCBC-MGㅜㅜ Tarcísio Versiani Azevedo Filho, TCBC-MG²; Domingos André Fernandes Drumond, TCBC-MG ${ }^{3}$; Pablo Acácio Oliveira Nunes Leal ${ }^{4}$}

\section{INTRODUÇÃO}

O rim é lesado em $51 \%$ dos traumas urogenitais, sendo que as lesões do parênquima são as mais frequentes. A ruptura da pelve renal é considerada incomum ${ }^{1} \mathrm{e}$, muitas vezes, diagnosticada e tratada tardiamente devido a sintomatologia frusta, principalmente se ocorre isoladamente. O objetivo do presente relato é apresentar as opções de diagnóstico e tratamento passíveis de serem utilizadas nessa curiosa lesão.

\section{RELATO DO CASO}

Paciente de 19 anos, masculino, vítima de queda de motocicleta, procurou assistência médica 30 horas após o trauma queixando-se de dor no flanco direito e na região lombar direita. Ao exame clínico apresenta como alteração apenas dor nos locais referidos. Foi realizado ultra-som de abdome que revelou presença de líquido laminar no espaço hepato-renal e hematoma peri-renal ipsilateral. A tomografia computadorizada (TC) do abdome mostrou grande extravasamento de contraste da pelve renal (Figura 1). O ureter direito não foi visualizado. Devido a esses achados e ao quadro clínico optou-se pelo tratamento cirúrgico. Após realização de laparotomia encontrou-se grande quantidade de urina no espaço retro-peritoneal decorrente de uma grande lesão da pelve renal. O parênquima renal e os vasos renais estavam íntegros. Realizou-se pieloplastia com pontos separados, nefrostomia com sonda de Foley $\mathrm{n}^{\circ} 18$ e drenagem laminar do retroperitônio. $\mathrm{O}$ paciente evoluiu bem, recebendo alta no quinto dia. No vigésimo-primeiro dia de pós-operatório realizou-se a pielografia com injeção de contraste pela nefrostomia (Figura 2) que revelou contrastação adequada do sistema pielocalicial e ureter, sem extravasamento. Em seguida foi retirada a nefrostomia. O paciente se encontra em controle ambulatorial, assintomático.

\section{DISCUSSÃO}

A ruptura isolada de pelve renal ocorre principalmente após trauma contuso e é considerada muito rara. Ela é

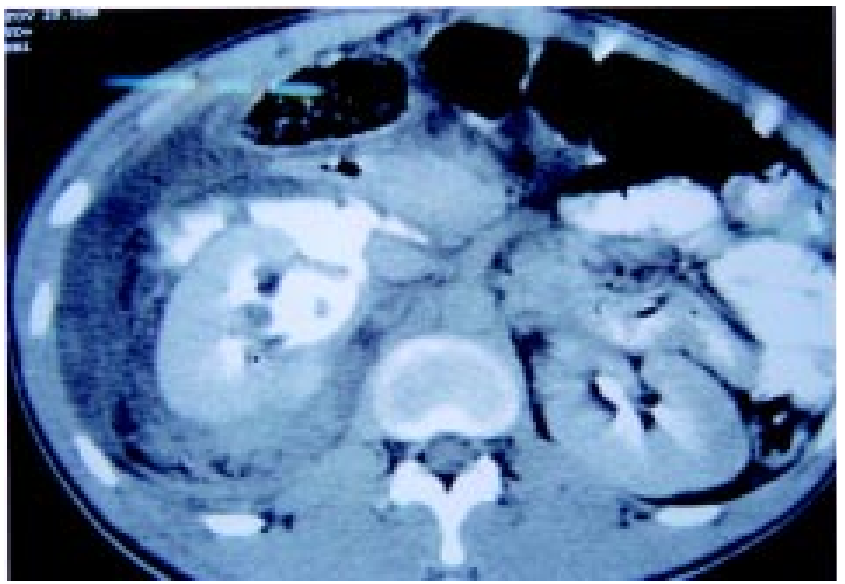

Figura 1 - Tomografia computadorizada do abdome mostrando extravasamento do meio de contraste na face medial do rim direito.

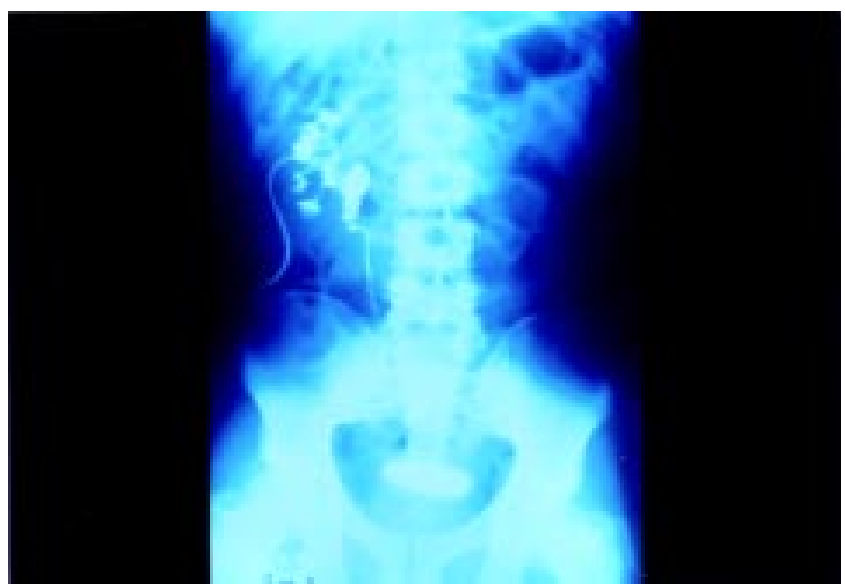

Figura 2 - Pielografia realizada através da nefrostomia mostrando sistema calicial, pelve renal e ureter bem contrastados e sem extravasamento.

excepcional se não houver variação anatômica, anomalia ou doença associada do sistema coletor. Ocorre mais frequentemente em crianças e à direita. A presença de lesões associadas dificulta ainda mais o diagnóstico por desviar a atenção do médico para o tratamento daquelas mais graves e por retar-

\footnotetext{
1. Cirurgião Titular do Hospital João XXIII (FHEMIG); Cirurgião do Hospital Evangélico. Belo Horizonte. MG; Instrutor do ATLS-MG.

2. Cirurgião Titular do Hospital João XXIII (FHEMIG); Cirurgião do Hospital SOCOR-Belo Horizonte. MG.

3. Chefe do Serviço de Cirurgia Geral e do Trauma do Hospital João XXIII(FHEMIG); Cirurgião do Hospital Felício Rocho - Belo Horizonte. MG; State Faculty do Núcleo ATLS-MG.

4. Cirurgião Substituto Cirurgia Geral e do Trauma do Hospital João XXIII(FHEMIG). Belo Horizonte. MG.
}

Recebido em 08/08/2005

Aceito para publicação em 10/10/2005

Conflito de interesses: nenhum

Fonte de financiamento: nenhuma

Trabalho realizado no Hospital João XXIII; Fundação Hospitalar do Estado de Minas Gerais, Belo Horizonte - MG 
dar a realização de exames complementares devido à instabilidade hemodinâmica do paciente ${ }^{1}$. Muitas vezes o trauma da pelve renal passa despercebido até mesmo durante o ato operatório ${ }^{2}$.

Os principais mecanismos envolvidos na gênese do trauma da pelve renal são compressão ou esmagamento devido ao impacto do objeto contundente (volante, painel, guidom de bicicleta etc.), deslocamento desigual, por desaceleração, de partes mais ou menos fixas do órgão e aumento súbito da pressão intraluminal, grande o suficiente para vencer a resistência da parede da pelve renal. Qualquer condição patológica prévia que leve a sua dilatação, como hidronefrose, predispõe a sua ocorrência ${ }^{3,4}$.

A sintomatologia é pobre, caracterizada, principalmente, por dor lombar persistente. A presença de hematúria não é frequente. A análise do mecanismo de trauma é fator fundamental para o diagnóstico. Fratura das ultimas costelas e da coluna toraco-lombar chamam a atenção para sua presença.

Os exames contrastados de imagem (urografia excretora e TC) confirmam o diagnóstico na maioria das vezes. A realização de imagens tardias é importante. A TC, na atualidade, é considerada o melhor exame porque além de diagnosticar a lesão, a sua magnitude, aumenta a acurácia da avaliação abdominal para possíveis lesões associadas ${ }^{5}$. Deve ser realizada em todo paciente com suspeita de lesão do trato urinário cujo mecanismo de trauma envolve desacelaração².

Os achados tomográficos que sugerem lesão isolada de pelve renal são: a) ausência de lesão do parênquima renal; b) presença de anomalia renal já existente; c) extravasamento do meio de contraste que pode ser medial ou circunferencial ao rim e d) contrastação ou não do ureter ipsilateral ${ }^{5}$.

Em serviços que não dispõem de TC a urografia excretora deve ser realizada. pois revela os mesmos achados da $\mathrm{TC}^{3}$. A pielografia retrógrada pode ser utilizada, em casos selecionados, quando se suspeita de avulsão completa do ureter $^{3,5}$.

O tratamento preconizado para a lesão de pelve renal é cirúrgico e consiste na realização de uma pielorrafia ou pieloplastia, dependendo da sua extensão. Recomenda-se associar uma descompressão do sistema coletor através de stent (duplo J) ou de nefrostomia, cujo objetivo é evitar uma hipertensão na pelve renal e deiscência da sutura ${ }^{3-5}$. Alguns autores recomendam, desde que a lesão e o extravasamento de contraste sejam pequenos, o tratamento endoscópico através de um posicionamento de um duplo J, que deve permanecer no local por um período de seis a oito semanas ${ }^{1,5}$.

$\mathrm{O}$ reconhecimento tardio da ruptura da pelve renal está associado com altas taxas de nefrectomia. Por outro lado, pacientes operados precocemente tem melhor prognóstico ${ }^{2}$.

\begin{abstract}
Blunt rupture of the renal pelvis is a exceedingly rare injury. Hematuria is absent in one-third of cases. Such injuries are usually caused by desacceleration forces. Despite improved imaging techniques, diagnosis of this injuries is frequently delayed. Diagnosis is suggested by the presence of massive medial extravasation of contrast material at excretory urography or computed tomography. Surgical treatment consists in pyeloplasty or pyeloraphy with stent placement (double J) or nephrostomy (Rev. Col. Bras. Cir. 2007; 34(3): 208-209).
\end{abstract}

Key words: Abdominal injuries; Rupture; Kidney pelvis.

\title{
REFERÊNCIAS
}

1. Powell MA, Nicholas JM, Davis JW. Blunt ureteropelvic junction disruption. J Trauma. 1999:47(1):186-8.

2. Boone TB, Gilling PJ, Husmann DA. Ureteropelvic junction disruption following blunt abdominal trauma. J Urol. 1993;150(1):33-6.

3. Hopkins TB, Klein LA. Disruption of the renal pelvis by blunt trauma. J Trauma. 1975;15(3):250-4.

4. Bogdanovic J, Djozic J, Idjuski S, Popov M, Sekulic V, Stojkov J. Successful surgical reconstruction of ruptured renal pelvis following blunt abdominal trauma. Urol Int. 2002;68(4):302-4.

5. Kawashima A, Sandler CM, Corriere JN Jr, Rodgers BM, Goldman SM. Ureteropelvic junction injuries secondary to blunt abdominal trauma. Radiology. 1997;205(2):487-92.
Como citar este artigo:

Starling SV, Azevedo Filho TV, Drumond DAF, Leal PAO. Ruptura isolada da pelve renal secundária a trauma abdominal contuso. Rev Col Bras Cir. [periódico na Internet] 2007; 34(2). Disponível em URL: http://www.scielo.br/rcbc

Endereço para correspondência:

Sizenando Vieira Starling

Rua Grão Mogol, 698 apto 304

Sion

30310-010 - Belo Horizonte - MG

E-mail: sizastarling@bol.com.br 